sinnigen und klugen Analysen von Rainer Holtschneider und Volker Ratzmann. Beide machen deutlich, wie gewinnbringend Einschätzungen von an solchen Reformprozessen unmittelbar Beteiligten sein können

Gerade multidisziplinär angelegte Sammelbände stellen für Herausgeber eine besondere Herausforderung dar. Um trotz der Vielfalt an Perspektiven und Herangehensweisen ein gewisses Maß an innerer Kohärenz zu erreichen, bedarf es einer sorgsamen Konzeptionalisierung sowie einleitender und abschließender Kapitel, die dem Leser einen roten Faden vermitteln, Ergebnisse zusammentragen und einordnen. Neben den beachtlichen Qualitätsunterschieden der einzelnen Beiträge liegt in der völlig unzureichenden editorischen Bearbeitung das zweite grundsätzliche Problem des Bandes. So erscheint die Grobgliederung in sechs Bereiche willkürlich und ist nur schwer nachvollziehbar. Einleitende und abschließende Beiträge fehlen völlig, sieht man von einem kurzen Vorwort ab. Obwohl der erste Artikel von Ralf Thomas Baus und Udo Margedant eine gute Einordnung des Standes der Föderalismusreform II vornimmt, kann er dieses Manko allein auch nicht auffangen, zumal er völlig abrupt und ohne Zusammenfassung nach einer kurzen Diskussion ausgewählter Inhalte der Reform aufhört. Obwohl der Untertitel des Bandes eine Einschätzung der Föderalismusreform II vor dem Hintergrund internationaler Erfahrungen verspricht, weisen von den insgesamt 25 Beiträgen lediglich zehn - im weitesten Sinne - einen Bezug zur europäischen Ebene oder zu anderen Ländern auf. Auch das zweite Ziel des Bandes, eine zu sehr an technischen Details orientierte Diskussion zu vermeiden, wird nicht durchgängig erreicht, was beispielsweise an einem Text mit dem ominösen Titel „Die Informationstechnik ist das Betriebssystem der deutschen Verwaltung“ deutlich wird.

Dem an der Reform der bundesstaatlichen Ordnung in Deutschland interessierten Leser wird also bei der Lektüre des Buches einiges abverlangt, denn es handelt sich dabei letztlich um eine weitgehend unsystematische Sammlung von Beiträgen, die oftmals kaum über das Niveau von Diskussionspapieren hinauskommen. Dies wäre kaum zu bemängeln, wenn die Konferenzdokumentation als Graue Literatur publiziert worden wäre. Eine Veröffentlichung für knapp 60 Euro bei einem renommierten Verlag lässt sich in dieser Form hingegen nur schwer rechtfertigen.

Jörg Broschek

\title{
Schlaglichter in schattigen Verwaltungskorridoren
}

Döhler, Marian: Die politische Steuerung der Verwaltung. Eine empirische Studie über politisch-administrative Interaktionen auf der Bundesebene, Nomos Verlagsgesellschaft, Baden-Baden 2007, 380 Seiten, €69,-.

Spätestens seit Max Weber gilt die öffentliche Verwaltung in Deutschland als ein Sinnbild wohlgeordneter Strukturen: Feste Hierarchien, ausgeprägtes Fachwissen und große personelle Konstanz sind ihr zueigen. Umso mehr erstaunt es immer wieder, wie viele Lücken die Kenntnis über die Verwaltung in Deutschland hat: Schon ihre Größe - zum Beispiel die Anzahl der Behörden und ihrer Beschäftigten - kann nur geschätzt werden. Dies hängt eng zusammen mit dem sehr ausgeprägten Ressortprinzip und der föderalen Ordnung der Bun- 
desrepublik, in der grundsätzlich die Länder für die Durchführung der Verwaltungsaufgaben verantwortlich sind. Nur ausnahmsweise und für eng begrenzte Themenbereiche gibt es Einrichtungen der Bundesverwaltung; entstanden sind zudem eine Reihe von komplizierten Konstruktionen wie die Auftragsverwaltung im Straßenbau und die Organleihe im Hochbau des Bundes. In den 1990er Jahren sind überdies zahlreiche organisatorische Neuaufstellungen in Form von Körperschaften, Stiftungen und Anstalten von Bund und Ländern vorgenommen worden, die zu allem Überfluss auch noch unsystematisch benannt wurden.

Die Habilitationsschrift von Marian Döhler bringt etwas Licht in die schattigen Verwaltungskorridore des Bundes. Untersucht wird hier das Zusammenspiel von Ministerien und ihnen nachgeordneten Behörden, indem exemplarisch das Bundesaufsichtsamt für das Versicherungswesen, das Bundeskartellamt, das Umweltbundesamt und das Bundesgesundheitsamt (beziehungsweise nach dessen Aufspaltung: das Bundesinstitut für Arzneimittel und Medizinprodukte) betrachtet werden. Insbesondere geht es um die Frage, ,auf welche Art und Weise und mit welcher Intensität die politische Steuerung von Bundesbehörden durch die Ministerialverwaltung stattfindet" (S. 21). Vielfältige Erkenntnisse aus der deutschen und angelsächsischen Verwaltungs- und Organisationswissenschaft werden dabei berücksichtigt. Neben der ausführlichen Behandlung der empirischen Beispiele gibt Döhler einen Überblick zu den Grundlagen des deutschen Verwaltungsmodells und beschäftigt sich mit Wandlungsprozessen hierarchischer Steuerung sowie den eingesetzten Steuerungsinstrumenten.

Die Untersuchung der konkreten Fälle erfolgte anhand eines einheitlichen Rasters, mit dem zunächst für alle genannten Behörden das jeweilige Politikfeld skizziert, sodann die Genese sowie Struktur der Einrichtungen und die Interaktion mit dem jeweils übergeordneten Ministerium dargestellt wird. Neben der Auswertung vielfältiger schriftlicher Quellen stützt sich die Analyse auf 34 Experteninterviews mit aktiven und ehemaligen Beschäftigten der genannten Behörden.

Nach dem vom Bundesverfassungsgericht festgeschriebenen Modell ist das Verwaltungshandeln durch ununterbrochene Legitimationsketten zum demokratischen Wahlakt rückzukoppeln. An der Schnittstelle zwischen Ministerien und Bundesoberbehörden wird dies mit verschiedenen Formen der Aufsicht (wie Fach-, Dienst- und Rechtsaufsicht) sichergestellt. Was genau der Aufsicht unterliegt, ist in der Realität allerdings häufig umstritten, und wer sich mit Verwaltungspraktikern einmal über die Definition der verschiedenen Formen auseinandergesetzt hat, bemerkt schnell, dass es hiervon sehr vielfältige Verständnisse gibt. In diesem Zusammenhang stellt Döhler in der empirischen Untersuchung fest, dass das mit der Fachaufsicht verbundene ministerielle Weisungsrecht nicht nur „möglichst geräuschlos" (S. 222), sondern überhaupt nur sehr zurückhaltend genutzt wird. Wirkung entfaltet das Instrument gerade durch sein Drohpotenzial. Die Assoziation mit dem in einem anderen Zusammenhang geprägten Begriff von der Problemlösung im „Schatten der Hierachie" (Fritz W. Scharpf) liegt nahe, doch Döhler warnt in seinen Schlussbetrachtungen ausdrücklich vor der unreflektierten Übertragung entsprechender Diagnosen: „Die verbreitete Rede von einer 'neuen Staatlichkeit', die sich durch eine kooperative bis konsensuale Form des Umgangs mit den Adressaten auszeichnet, sollte im Bereich inneradministrativer Beziehungen mit Vorsicht verwendet werden" (S. 319).

Klar wird zudem, dass die Gestaltung von Aufbau und Geschäftsprozessen der nachgeordneten Behörden die aufsichtführenden Ministerien nur wenig interessiert (S. 233), wäh- 
rend insbesondere für Publikationen durchaus häufig ein Erlaubnisvorbehalt gesetzt wird. Ein zentrales Fazit der Untersuchung ist also, dass politische Steuerung der Ministerien nicht nur meist fernab der Öffentlichkeit, sondern oftmals auch vorrangig implizit erfolgt (S. 238). Dennoch werden weitgehende ministerielle Eingriffsrechte bei der Neugestaltung von nachgeordneten Behörden weiterhin routinemäßig vorgesehen - sie dienen nach Döhler „der präventiven Sicherung zukünftiger Hierarchieverwendungsrechte“ (S. 246).

Die Untersuchung zeigt eindrucksvoll, wie ausdauerndes, gut informiertes „Nachbohren “ in speziellen Politikfeldern zu einem realistischen und empirisch gesättigten Bild des täglichen Geschäfts öffentlicher Verwaltung führen kann. Die in dieser Arbeit angesprochenen Nuancen der Wirkung von ministerieller Steuerung wären bei Beschränkung auf eine formale Herangehensweise kaum sichtbar zu machen. Das Buch ist im besten Sinne ein interdisziplinäres Werk, das vielfältige theoretische Ansätze aufnimmt, sie vor dem Hintergrund der Empirie reflektiert und in angemessener Abwägung und Vorsicht zu klaren Ergebnissen kommt. Der verwaltungswissenschaftliche Blick des Verfassers berücksichtigt zwar Erkenntnisse aus der Parlamentarismusforschung nur am Rande - und er scheint an zwei Stellen sogar die Ministerien mit der Funktion der „Gesetzgebung“ (S. 240, S. 251) zu betrauen -, doch ohne Frage ist ein solch differenzierter Blick auf die Mechanismen von Steuerung, Aufsicht und Kontrolle nicht nur für das Verhältnis zwischen Ministerien und ihnen nachgeordneten Behörden angezeigt, sondern dürfte auch für die Beziehungen zwischen Regierung und Parlament instruktiv sein.

Sven T. Siefken

\section{Wer war das Volk? Neue Ansätze zur Erforschung der Weimarer Verfassung}

Bollmeyer, Heiko: Der steinige Weg zur Demokratie. Die Weimarer Nationalversammlung zwischen Kaiserreich und Republik, Campus Verlag, Frankfurt am Main / New York 2007, 476 Seiten, € 49,90.

Die Weimarer Republik und ihre Verfassung sind seit einigen Jahren aus dem Schatten ihres Endes herausgetreten. Viel zu lange galt unter Historikern die Devise Karl Dietrich Erdmanns, wonach alle Forschungen zur ersten Demokratie „mit Notwendigkeit - ausgesprochen oder unausgesprochen - unter der Frage nach den Ursachen ihres Zusammenbruchs“ stehen. Zwar war dies mit Blick auf den Horror des Nachfolgenden nicht unverständlich; wenn dabei aber allzu einseitig auf die Verfassung, ihre vermeintlichen Schwächen und Geburtsfehler abgestellt wurde, hatte dies auch einen apologetischen Charakter: Das Versagen der Verfassung relativierte jenes einzelner politischer Akteure. Inzwischen hat sich der Blick auf die Weimarer Verfassung verändert, statt ihres Scheiterns sind ihre Entstehung, ihre Chancen und ihr Beitrag zur Demokratiegeschichte ins Zentrum der Aufmerksamkeit gerückt. Dabei interessieren vor allem die Demokratievorstellungen bei der Verfassungsgebung, und diesem Thema ist auch die geschichtswissenschaftliche Dissertation von Heiko Bollmeyer gewidmet, die in Bielefeld von Heinz Gerhard Haupt und Christoph Gusy betreut worden ist. 